\title{
Multifarious Chaotic Attractors and Its Control in Rigid Body Attitude Dynamical System
}

\author{
Yang Wang $\left(\mathbb{D},{ }^{1}\right.$ Zhen Wang $\mathbb{D},{ }^{1}$ Dezhi Kong $\mathbb{D},{ }^{2}$ Lingyun Kong $\mathbb{D}{ }^{1}$ and Yukun Qiao $\mathbb{D}^{1}$ \\ ${ }^{1}$ School of Science, Xijing University, Xi'an 710123, China \\ ${ }^{2}$ School of Mechanical Engineering, Northwestern Polytechnical University, Xi'an 710072, China \\ Correspondence should be addressed to Zhen Wang; williamwangz@yeah.net
}

Received 27 May 2020; Revised 21 October 2020; Accepted 30 October 2020; Published 21 November 2020

Academic Editor: Jose de Jesus Rubio

Copyright (c) 2020 Yang Wang et al. This is an open access article distributed under the Creative Commons Attribution License, which permits unrestricted use, distribution, and reproduction in any medium, provided the original work is properly cited.

The Euler dynamical equation which describes the attitude motion of a rigid body will exhibit very complex dynamic behaviors under the action of different external torques. Many special types of new chaotic attractors are presented, including hidden attractors, double-body-double-core chaotic attractors, and single-body-three-core-tree-wing chaotic attractors. The position of equilibrium points in several typical cases of the Euler dynamic equation is solved, and the stability of linearized equation at each equilibrium point and its influence on the formation of the chaotic attractor are analyzed. An improved nonlinear relay control law based on Euler angle feedback is developed to stabilize a new chaotic spacecraft attitude motion to an appointed equilibrium point or a periodic orbit.

\section{Introduction}

The detailed exploration of the chaotic attitude motion of spacecraft and satellites still remains one of the main problems of rigid body attitude dynamics. A number of investigators have demonstrated that spinning satellite [1], dual-spin spacecraft (DSSC) [2-4], multispin spacecraft (MSSC) [5], gyrostat satellite [6-12], tethered satellite, and other complicated satellites would exhibit chaotic attitude motion in gravitational fields [13], geomagnetic field [14, 15], and sunlight flux. Beletsky et al [14] discussed the chaotic motion of a magnetic spacecraft in circular polar orbit without damping and gravitational torque. Chen and Liu $[15,16]$ investigated chaotic attitude motion of a magnetic rigid spacecraft with internal damping in a circular orbit near the equatorial plane of the earth. These works mainly analyzed the dynamics of different mathematical models of rigid body attitude motion from a different point of view.

The attitude motion of a rigid body could be described by the Eulerian dynamic equations. This nonlinear equation will present very complex dynamic behaviors, including various forms of chaotic motion. Leipnik and Newton [17] found two strange attractors from this system. In the previous paper $[18,19]$, we introduced several new chaotic attractors but did not analyze the influence of the properties of equilibrium point on the formation of chaotic attractors.

Different control techniques have been employed to suppress or manipulate chaotic attitude motion of spacecraft and satellites, including sliding mode variable structure control [20,21], time-delayed feedback control [22, 23], observer-based control [24, 25], impulsive control, adaptive control, and open-plus-closed-loop control. Alban and Antonia [26] made use of three methods to control a sixdimensional chaotic system that describes the attitude dynamics of a rigid body spacecraft subjected to deterministic external perturbations that induce chaotic motion when no control is acted. The three techniques are a simple delayed feedback control method, the Otani-Jones technique, and a higher dimensional variation of the OGY method. Other control methods were employed to suppress chaos by Meehan and Asokanthan [1] and Awad [20]. Generally, the angular velocity of the spacecraft $\omega$ was chosen as feedback control variable. However, $\omega$ can be measured directly in practice.

Different spacecraft have different mission requirements and need different control methods. A spinning spacecraft usually is required to rotate along one axis with constant 
rotation rate and not to rotate along the other two axes. When a spinning spacecraft is disturbed and produces unexpected rotation, the nozzle thruster is usually used to control it. Due to the coupling effect of the three channels, the single-channel dead zone relay control method cannot effectively suppress a chaotic motion of spinning spacecraft.

The innovation of this paper lies in the following:

(1) Many special types of new chaotic attractors are presented, including hidden attractors, doublebody-double-core chaotic attractors, and singlebody-three-core-tree-wing chaotic attractors.

(2) The position of equilibrium points in several typical cases of the Euler dynamic equation is solved, and the stability of linearized equation at each equilibrium point and its influence on the formation of chaotic attractor are analyzed.

(3) A more practicable and efficient control techniques are utilized to suppress chaos and control state of the system to an appointed fixed point or a periodic orbit.

\section{The Attitude Motion Equations of a Rigid Body}

The attitude motion of a rigid body could be described by the Eulerian equations, which consists of kinematic equations and dynamic equations.

The attitude orientation of spacecraft at a given point can be locally described in terms of three Eulerian angles $\varphi, \theta$, and $\psi$ which are successive clockwise rotations about inertial axes $X, Y$, and $Z$, respectively. These successive rotations transform the inertially fixed set of orthonormal axes $X, Y$, and $Z$ (regarded as initially instantaneously coincident with the body axes) into the axes $x, y$, and $z$ fixed in the body. The kinematic equation of the rigid body can be expressed as [21]

$$
\left\{\begin{array}{l}
\omega_{x}=\dot{\varphi} \cos \psi \cos \theta+\dot{\theta} \sin \psi, \\
\omega_{y}=-\dot{\varphi} \sin \psi \cos \theta+\dot{\theta} \cos \psi, \\
\omega_{z}=\dot{\psi}+\dot{\varphi} \sin \theta,
\end{array}\right.
$$

or the following form

$$
\left\{\begin{array}{l}
\dot{\varphi}=\frac{\left(\omega_{x} \cos \psi-\omega_{y} \sin \psi\right)}{\cos \theta}, \\
\dot{\theta}=\omega_{x} \sin \psi+\omega_{y} \cos \psi, \\
\dot{\psi}=\omega_{z}-\left(\omega_{x} \cos \psi-\omega_{y} \sin \psi\right) \tan \theta .
\end{array}\right.
$$

Here, $\omega=\left(\omega_{x}, \omega_{y}, \omega_{z}\right)$ are the angular velocities of a rigid body. The dynamical motion equations of a spacecraft with principle axes at the center of mass are [21]

$$
\left\{\begin{array}{l}
I_{x} \dot{\omega}_{x}=\omega_{y} \omega_{z}\left(I_{y}-I_{z}\right)+M_{x}+u_{x}, \\
I_{y} \dot{\omega}_{y}=\omega_{x} \omega_{z}\left(I_{z}-I_{x}\right)+M_{y}+u_{y}, \\
I_{z} \dot{\omega}_{z}=\omega_{y} \omega_{x}\left(I_{x}-I_{y}\right)+M_{z}+u_{z} .
\end{array}\right.
$$

Here, $I_{x}, I_{y}$, and $I_{z}$ are the principal moments of inertia with respect to body axes $x, y$, and $z ; u_{x}, u_{y}$, and $u_{z}$ are the component of the control torques; and $M_{x}, M_{y}$, and $M_{z}$ are the perturbing torques. The total disturbing torque $M$ which is put on a spacecraft can be written as

$$
M=M_{g}+M_{m}+M_{c}+M_{f}+M_{d}+L .
$$

Here, $M_{g}$ and $M_{m}$ are the gravitational and magnetic torque; $M_{d}$ is the internal damping torque which is proportional to the angular velocity of the body with coefficient c; $M_{c}$ and $M_{f}$ are atmosphere resistance torque and solar pressure torque. They all have relation to the attitude angles $\varphi, \theta$, and $\psi$, the attitude angular velocities $\omega$, and the orbital angular velocities $\omega_{0}$. For example, the component of gravitational torque $M_{g z}$ on $Z$-axis can be written as

$$
M_{g z}=-\omega_{0}^{2}\left(I_{y}-I_{z}\right) \sin \varphi \cos \varphi .
$$

The magnetic torque about $\mathrm{Z}$-axis can be obtained as

$$
M_{m z}=k \sin \alpha[3 \cos (\varphi-\beta-\gamma)-\cos (\varphi+\beta+\gamma)] .
$$

Here, $k$ is a coefficient with respect to the magnetic moment constant of the earth and the magnetic moment of the spacecraft and the distance between spacecraft and the earth. $\alpha$ is the angle of inclination of orbital plane. $\beta$ is the argument of perigee. $\gamma$ is the true anomaly of spacecraft as the angular coordinate measured from perigee.

Equation (3) can be rewritten as

$$
\dot{\vec{\omega}}=\left[a_{x} \omega_{y} \omega_{z} a_{y} \omega_{x} \omega_{z} a_{z} \omega_{x} \omega_{y}\right]^{T}+\vec{A} \vec{\omega}+\dot{\vec{\omega}}_{u} .
$$

Here, $a_{x}=\left(I_{y}-I_{z}\right) / I_{x}, a_{y}=\left(I_{z}-I_{x}\right) / I_{y}, \quad a_{z}=\left(I_{x}-\right.$ $\left.I_{y}\right) / I_{z}, \quad \vec{\omega}=\left[\begin{array}{lll}\omega_{x} & \omega_{y} & \omega_{z}\end{array}\right]^{T}, \dot{\vec{\omega}}_{u}=\left[\begin{array}{lll}u_{x} / I_{x} & u_{y} / I_{y} & u_{z} / I_{z}\end{array}\right]^{T}$, $\vec{A} \vec{\omega}=\left[\begin{array}{lll}M_{x} / I_{x} & M_{y} / I_{y} & M_{z} / I_{z}\end{array}\right]^{T}, \quad \vec{A}=\left[a_{i j}\right] \in R^{3 \times 3}(i, j=$ $1,2,3)$ is a perturbing frequency matrix.

\section{Multifarious Chaotic Attractors}

Equation (7) is a more generalized 3-dimensional nonlinear system which will exhibit complex (periodic, quasiperiodic, or chaotic) dynamic behaviors under the action of different external torques.

3.1. Well-Known Chaotic Attractors in This System. A spinning rigid spacecraft is symmetrical with respect to the body axes $O x$, i.e., $I_{y}=I_{z}$. In general, it is appropriate to choose $I_{x}=2 I_{y}=2 I_{z}$ for stabilizing the spinning motion of the spacecraft, i.e., $\left(a_{x}, a_{y}, a_{z}\right)=(0,-1,1)$. When $u_{x}=u_{y}=$

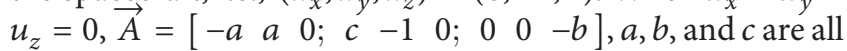
perturbing frequency parameters, and equation (7) is equal to the normal Lorenz equations [22]:

$$
\left\{\begin{array}{l}
\dot{\omega}_{x}=-a \omega_{x}+a \omega_{y} \\
\dot{\omega}_{y}=c \omega_{x}-\omega_{y}-\omega_{x} \omega_{z} \\
\dot{\omega}_{z}=-b \omega_{z}+\omega_{x} \omega_{y} .
\end{array}\right.
$$


It will exhibit chaotic motion when $(a, b, c)=(10,-8 / 3$, $28)$ as we all know. When $\left(a_{x}, a_{y}, a_{z}\right)=(10,-5,5), u_{x}=$

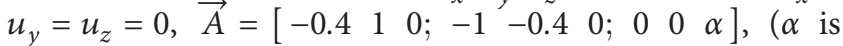
alterable parameter), system (7) is a chaotic system named the Leipnik-Newton system [22, 23]:

$$
\left\{\begin{array}{l}
\dot{\omega}_{x}=-a \omega_{x}+\omega_{y}+10 \omega_{y} \omega_{z} \\
\dot{\omega}_{y}=-\omega_{x}-0.4 \omega_{y}+5 \omega_{x} \omega_{z} \\
\dot{\omega}_{z}=b \omega_{z}-5 \omega_{x} \omega_{y} .
\end{array}\right.
$$

There are two strange attractors in this system when $a=$ 0.4 and $b=0.175$ as we all know.

Earlier papers [1] have taken $I_{x}=3, I_{y}=2, I_{z}=1$, $\left(a_{x}, a_{y}, a_{z}\right)=(1 / 3,-1,1)$,

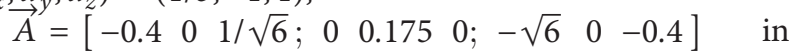
(7). These torques are chosen to be sufficiently large to induce chaotic motion and are comparable in magnitude with the available thruster torques. The dynamics of the satellite will then exhibit chaotic motion. We constructed the chaotic attractor of this system shown in Figure 1.

\subsection{New Chaotic Attractors}

3.2.1. A Type of Hidden Attractors. In the study of the chaotic motion of a nonlinear system, it is often concerned

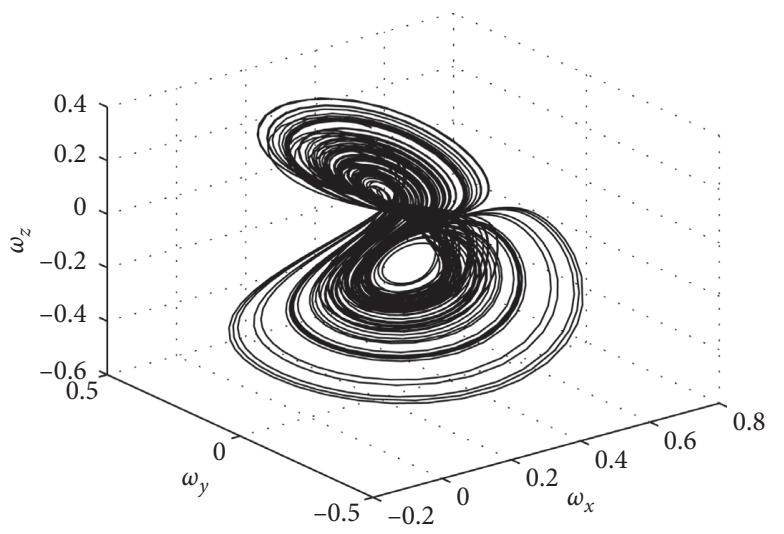

FIgURE 1: One chaotic attractor of system (7), starting point $(0.3694,0.0473,0.1105)$.

with the chaotic attractors near the equilibrium point. Some hidden attractors exist widely in the greater region or the smaller region of the phase space.

Let us take Leipnik-Newton system (9) as an example. There are five equilibriums in equation (9). The origin of coordinates $S_{0}:(0,0,0)$ is one of five equilibriums. The other four equilibriums are composed of the proper combination of the following three values:

$$
\begin{aligned}
& \omega_{x e 1,2,3,4}= \pm \sqrt{\frac{(4 a-15) b \pm b \sqrt{225-80 a}}{250 a}} \\
& \omega_{y e 1,2,3,4}= \pm \sqrt{\frac{(15-8 a) b \pm b \sqrt{225-80 a}}{200}} \\
& \omega_{z e 1,2,3,4}= \pm 5 \sqrt{\frac{(4 a-15) \pm \sqrt{225-80 a}}{250 a}} \cdot \frac{(15-8 a) \pm \sqrt{225-80 a}}{400}
\end{aligned}
$$

Example 1. Let $a=0.4$ and $b=0.3$, and the four equilibriums are

$$
\begin{aligned}
& S_{1}, S_{2}:( \pm 0.0413, \mp 0.1602,-0.1103), \\
& S_{3}, S_{4}:( \pm 0.3129, \mp 0.0403,0.2103) .
\end{aligned}
$$

The eigenvalues of the Jacobian linearization matrix at four equilibriums are the same: $S_{1}, S_{2}, S_{3}, S_{4}: \lambda_{1}=-0.8$, $\lambda_{2,3}=0.15 \pm 1.1419 i$. In the phase space, there exist one chaotic attractor near $S_{1}$ and $S_{2}$, and one periodic attractor near $S_{3}$ and $S_{4}$, and one big hidden attractor far away from equilibriums. The phase trajectories of the three attractors are shown in Figure 2. The starting point of the small periodic attractor is selected at $(-0.0459,0.0802,0.2871)$. The starting point of the big hidden attractor is selected at $(-74.2294,52.3574,-29.0246)$. Attractors. Investigating the reduced form of equation (7),

$$
\left\{\begin{array}{l}
\dot{\omega}_{x}=a_{x} \omega_{y} \omega_{z}+a_{11} \omega_{x} \\
\dot{\omega}_{y}=a_{y} \omega_{x} \omega_{z}+a_{22} \omega_{y} \\
\dot{\omega}_{z}=a_{z} \omega_{y} \omega_{x}+a_{33} \omega_{z}
\end{array}\right.
$$

When the feedback coefficients $a_{11}, a_{22}$, and $a_{33}$ make $0 \geq \mu_{k} \in R,(k=x, y, z)$, equation (12) degenerates to the single equilibrium point system. 


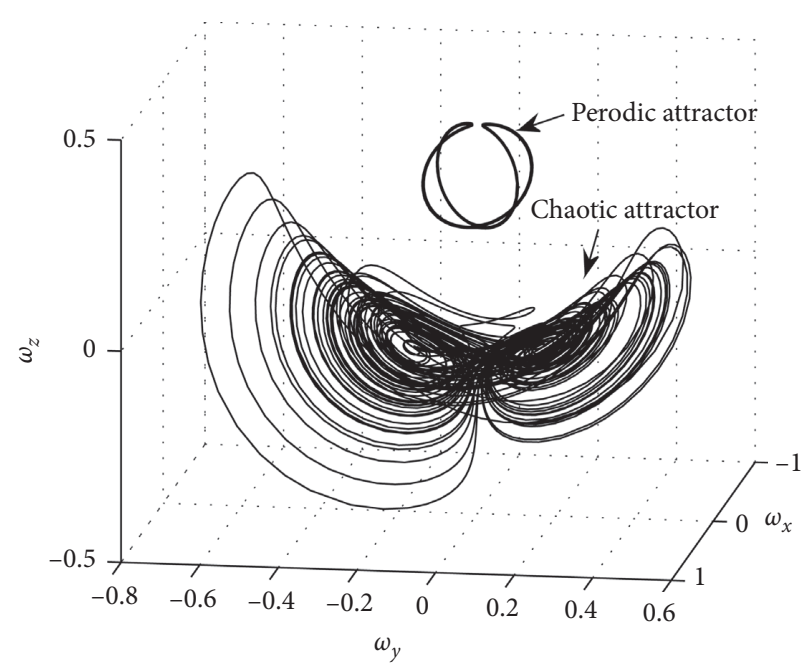

(a)

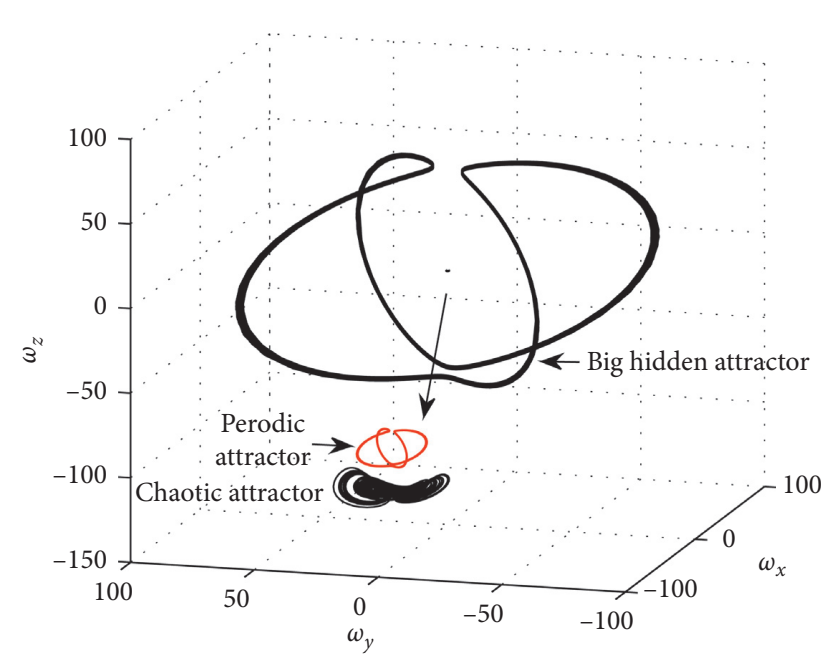

(b)

FIGURE 2: One chaotic attractor, one periodic attractor, and one big hidden attractor exist at the same phase space of system (9): (a) small periodic attractor; (b) big hidden attractor. $x, y, z)$.

Here, let us just discuss the case of $0 \leq \mu_{k} \in R$, ( $k=$

Let us assume $I_{x}>I_{y}>I_{z}>0, a_{x}>0, a_{y}<0, a_{z}>0$, and there are two cases of combination forms.

Case A: $a_{x}>0, a_{y}<0, a_{z}>0, a_{22}>0, a_{11}<0, a_{33}<0$. The other four equilibriums are expressed as

$$
\begin{aligned}
& S_{1}:\left(\mu_{x}, \mu_{y}, \mu_{z}\right), S_{2}:\left(\mu_{x},-\mu_{y},-\mu_{z}\right), \\
& S_{3}:\left(-\mu_{x},-\mu_{y}, \mu_{z}\right), S_{4}:\left(-\mu_{x}, \mu_{y},-\mu_{z}\right) .
\end{aligned}
$$

Case B: $a_{x}>0, a_{y}<0, a_{z}>0, a_{22}<0, a_{11}>0, a_{33}>0$. The other four equilibriums are expressed as

$$
\begin{aligned}
& S_{1}^{\prime}:\left(\mu_{x},-\mu_{y}, \mu_{z}\right), S_{2}^{\prime}:\left(\mu_{x}, \mu_{y},-\mu_{z}\right), \\
& S_{3}^{\prime}:\left(-\mu_{x}, \mu_{y}, \mu_{z}\right), S_{4}^{\prime}:\left(-\mu_{x},-\mu_{y},-\mu_{z}\right) .
\end{aligned}
$$

Equation (12) is linearized at equilibrium point $S_{1}:\left(\mu_{x}, \mu_{y}, \mu_{z}\right)$, and the Jacobian matrix is

$$
\vec{J}=\left[\begin{array}{ccc}
a_{11} & a_{x} \mu_{z} & a_{x} \mu_{y} \\
a_{y} \mu_{z} & a_{22} & a_{y} \mu_{x} \\
a_{z} \mu_{y} & a_{z} \mu_{x} & a_{33}
\end{array}\right] .
$$

The characteristic equation of the Jacobian linearization system is

$$
\lambda^{3}-\left(a_{11}+a_{22}+a_{33}\right) \lambda^{2}+4 a_{11} a_{22} a_{33}=0 .
$$

The eigenvalues can be calculated by the following steps:

$$
\begin{aligned}
\lambda_{1} & =u+v+\frac{a_{11}+a_{22}+a_{33}}{3}, \\
\lambda_{2,3} & =-\frac{u+v}{2}+\frac{a_{11}+a_{22}+a_{33}}{3} \pm i \frac{u-v}{2} \sqrt{3} .
\end{aligned}
$$

Here,

$$
\begin{aligned}
& u=\sqrt[3]{-\frac{q}{2}+\sqrt{Q}}, \\
& v=\sqrt[3]{-\frac{q}{2}-\sqrt{Q}}, \\
& Q=\left(\frac{p}{3}\right)^{3}+\left(\frac{q}{2}\right)^{2}, \\
& q=2\left(\frac{a_{11}+a_{22}+a_{33}}{3}\right)^{3}+4 a_{11} a_{22} a_{33}, \\
& p=-\frac{\left(a_{11}+a_{22}+a_{33}\right)^{2}}{3} .
\end{aligned}
$$

Simulation studies indicate that system (12) will exhibit complex dynamic behaviors under the different feedback coefficients.

When $a_{22}=\left|a_{11}-a_{33}\right|$, for example, $a_{11}=-1, a_{22}=2$, $a_{33}=-3$, there exists a single cycle attractor.

When $a_{22}>\left|a_{11}-a_{33}\right|$, for example, $a_{11}=-1, a_{22}=2$, $a_{33}=-2.8,-2.5,-2,-1.5$, system (12) exhibits a divergent trajectory, and the pace of trajectory divergence speeds up with the increases of the difference between $a_{11}$ and $a_{33}$.

When $a_{22}<\left|a_{11}-a_{33}\right|$, for example, $a_{11}=-1, a_{22}=2$, and $a_{33}=-3,-3.4,-3.42,-3.43,-3.44,-3.45 L \ldots$, system (12) exists, respectively, 1-cycle, 2-cycle, 3-cycle, 4-cycle, 6-cycle, 8-cycle attractor ... until the chaotic attractor.

Example 2. Let $a_{x}=0.25, a_{y}=-2 / 3, a_{z}=0.5, a_{11}=-1$, $a_{22}=2, a_{33}=-4$, and the other four equilibriums are

$$
\begin{aligned}
& S_{1}:(\sqrt{24}, \sqrt{32}, \sqrt{12}), \\
& S_{2}:(\sqrt{24},-\sqrt{32},-\sqrt{12}), \\
& S_{3}:(-\sqrt{24},-\sqrt{32}, \sqrt{12}), \\
& S_{4}:(-\sqrt{24}, \sqrt{32},-\sqrt{12}), \\
& \lambda_{1}=-4.5474, \\
& \lambda_{2,3}=0.7734 \pm 2.5374 i .
\end{aligned}
$$


The eigenvalues of the Jacobian linearization matrix at four equilibriums are the same: $\lambda_{1}=-4.5474, \lambda_{2,3}=$ $0.7734 \pm 2.5374 i$. There exist double-body-double-core chaotic attractors in the phase space. The phase trajectories of the two attractors are shown in Figure 3.

3.2.3. A Type of Single-Body-Three-Core Chaotic Attractors. Investigating another reduced form of equation (7),

$$
\left\{\begin{array}{l}
\dot{\omega}_{x}=a_{x} \omega_{y} \omega_{z}+a_{11} \omega_{x}+a_{12} \omega_{y}+a_{13} \omega_{z}, \\
\dot{\omega}_{y}=a_{y} \omega_{x} \omega_{z}+a_{22} \omega_{y}, \\
\dot{\omega}_{z}=a_{z} \omega_{y} \omega_{x}+a_{33} \omega_{z} .
\end{array}\right.
$$

There are five equilibriums in equation (13). The origin of coordinates $S_{0}:(0,0,0)$ is one of five equilibriums. The other four equilibriums are composed of the proper combination of the following values:

$$
\left\{\begin{array}{l}
\omega_{x e 1,2,3,4}= \pm \sqrt{\frac{a_{22} a_{33}}{a_{y} a_{z}}}, \\
\omega_{y e 1,2}=\frac{a_{12} a_{13}-a_{13} a_{z} \sqrt{a_{22} a_{33} / a_{y} a_{z}}}{2 a_{x} a_{z} \sqrt{a_{22} a_{33} / a_{y} a_{z}}} \pm \frac{\sqrt{\left(a_{12} a_{13}-a_{13} a_{z} \sqrt{a_{22} a_{33} / a_{y} a_{z}}\right)^{2}+4 a_{x} a_{11} a_{22} a_{33}^{2} / a_{y}}}{2 a_{x} a_{z} \sqrt{a_{22} a_{33} / a_{y} a_{z}}}, \\
\omega_{y e 3,4}=\frac{a_{12} a_{13}+a_{13} a_{z} \sqrt{a_{22} a_{33} / a_{y} a_{z}}}{-2 a_{x} a_{z} \sqrt{a_{22} a_{33} / a_{y} a_{z}}} \pm \frac{\sqrt{\left(a_{12} a_{13}+a_{13} a_{z} \sqrt{a_{22} a_{33} / a_{y} a_{z}}\right)^{2}+4 a_{x} a_{11} a_{22} a_{33}^{2} / a_{y}}}{-2 a_{x} a_{z} \sqrt{a_{22} a_{33} / a_{y} a_{z}}} \\
\omega_{z e 1,2}=\frac{a_{22} a_{13}-a_{12} a_{y} \sqrt{a_{22} a_{33} / a_{y} a_{z}}}{2 a_{x} a_{y} \sqrt{a_{22} a_{33} / a_{y} a_{z}}} \pm \frac{\sqrt{\left(a_{22} a_{13}-a_{12} a_{y} \sqrt{a_{22} a_{33} / a_{y} a_{z}}\right)^{2}+4 a_{x} a_{11} a_{33} a_{22}^{2} / a_{z}}}{2 a_{x} a_{y} \sqrt{a_{22} a_{33} / a_{y} a_{z}}} \\
\omega_{z e 3,4}=\frac{a_{22} a_{13}+a_{12} a_{y} \sqrt{a_{22} a_{33} / a_{y} a_{z}}}{-2 a_{x} a_{y} \sqrt{a_{22} a_{33} / a_{y} a_{z}}} \pm \frac{\sqrt{\left(a_{22} a_{13}+a_{12} a_{y} \sqrt{a_{22} a_{33} / a_{y} a_{z}}\right)^{2}+4 a_{x} a_{11} a_{33} a_{22}^{2} / a_{z}}}{-2 \sqrt{a_{22} a_{33} / a_{y} a_{z}}}
\end{array} .\right.
$$

If $a_{y}$ and $a_{22}$ are the opposite sign, then $a_{z}$ and $a_{33}$ must be the opposite sign. When $\omega_{x e}>0, \omega_{y e}$ and $\omega_{z e}$ must be the same sign. When $\omega_{x e}<0, \omega_{y e}$ and $\omega_{z e}$ must be the opposite sign.

Compared with equation (12), feedback control parameters $a_{12} \omega_{y}$ and $a_{13} \omega_{y}$ are applied to the system, the four equilibriums are no longer symmetric, and the dynamic properties of equation (13) near the four equilibriums present a complicated situation. When the real part of the eigenvalues of Jacobian linearization matrix at four equilibriums are greater than zero: $S_{1}, S_{2}, S_{3}, S_{4}: \operatorname{Re}\left(\lambda_{2,3}\right)>0$, the four equilibrium points are unstable saddle odd points, and the system will produce a variety of single-body-four-corefour-wing chaotic attractors. When one of the real part is less than zero: $S_{4}: \operatorname{Re}\left(\lambda_{2,3}\right)<0$, this equilibrium point is stable, and the system will produce a type of single-body-threecore-tree-wing chaotic attractor.
Example 3. Let $a_{x}=0.25, a_{y}=-2 / 3, a_{z}=0.5, a_{11}=-1$, $a_{22}=2, a_{33}=-4, a_{12}=2, a_{13}=2$, and the other four equilibriums are $S_{1}:(4.899,1.43,0.871), S_{2}:(4.899$, $-22.49,-13.77)$,

$S_{3}:(-4.899,8.729,-5.346)$, and $S_{4}:(-4.899,-3.666,2.245)$.

The eigenvalues of the Jacobian linearization matrix at four equilibriums are $S_{1}: \lambda_{1}=-3.7695, \lambda_{2,3}=0.3848 \pm$ $2.0893 i, S_{2}: \lambda_{1}=-9.0734, \lambda_{2,3}=3.0367 \pm 4.5174 i, S_{3}: \lambda_{1}=$ $-6.8623, \lambda_{2,3}=1.9312 \pm 2.0383 i, \quad S_{4}: \lambda_{1}=-2.9554, \lambda_{2,3}=$ $-0.0223 \pm 2.7725 i$.

Obviously, $S_{1}, S_{2}, S_{3}: \operatorname{Re}\left(\lambda_{2,3}\right)>0$ but $S_{4}: \operatorname{Re}\left(\lambda_{2,3}\right)<0, S_{4}$ is stable equilibrium point, and $S_{1}, S_{2}, S_{3}$ are unstable. There is a small attraction basin around $S_{4}$ (shown in Figure 4(a)) and a single-body-three-core-tree-wing chaotic attractor which are formed by $S_{1}, S_{2}$, and $S_{3}$ in the larger domain. The phase trajectories of the attractors and the attraction basin (red point) are shown in Figure 4. 


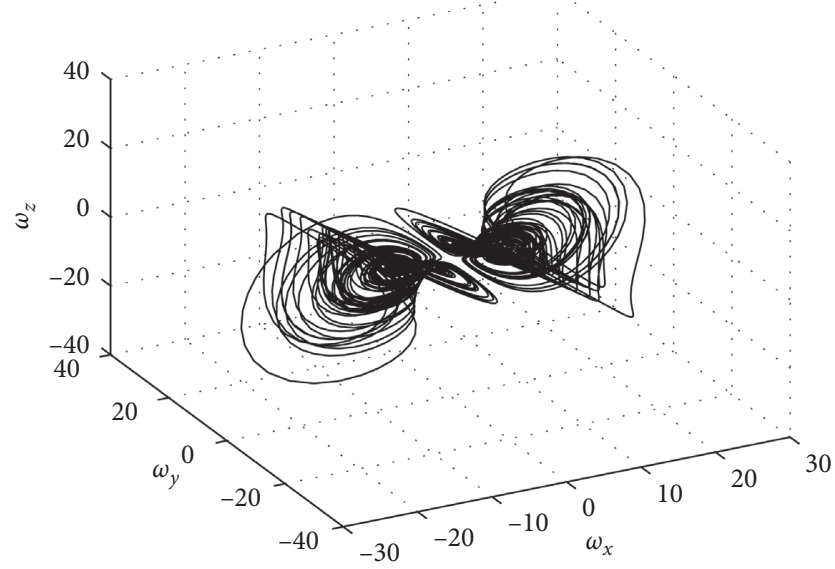

(a)

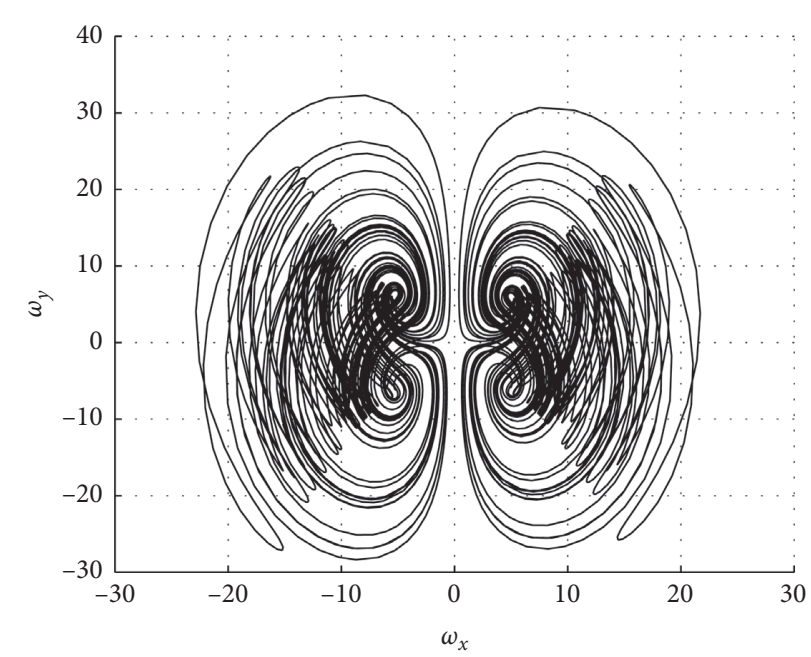

(b)

Figure 3: One double-body-double-core chaotic attractor of system (12): (a) chaotic attractor; (b) the projection on the $O \omega_{x} \omega_{y}$ plane.

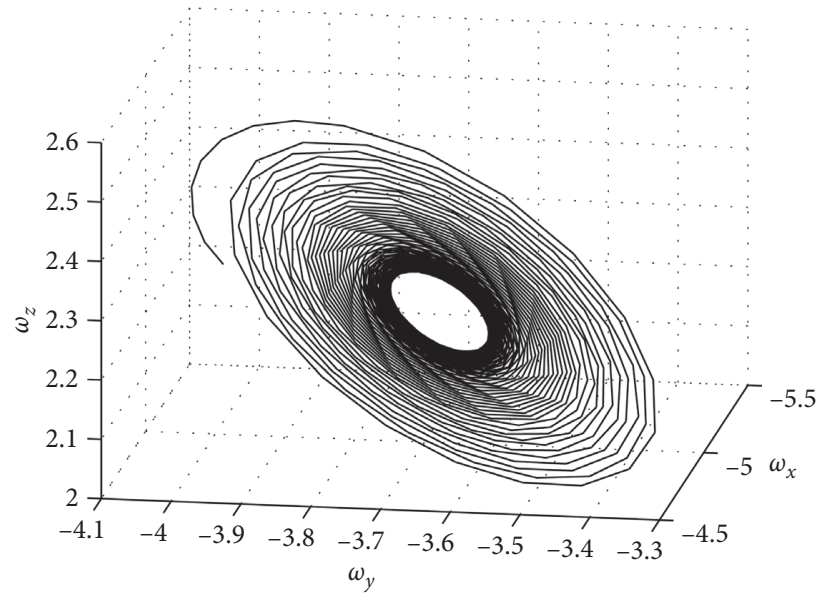

(a)

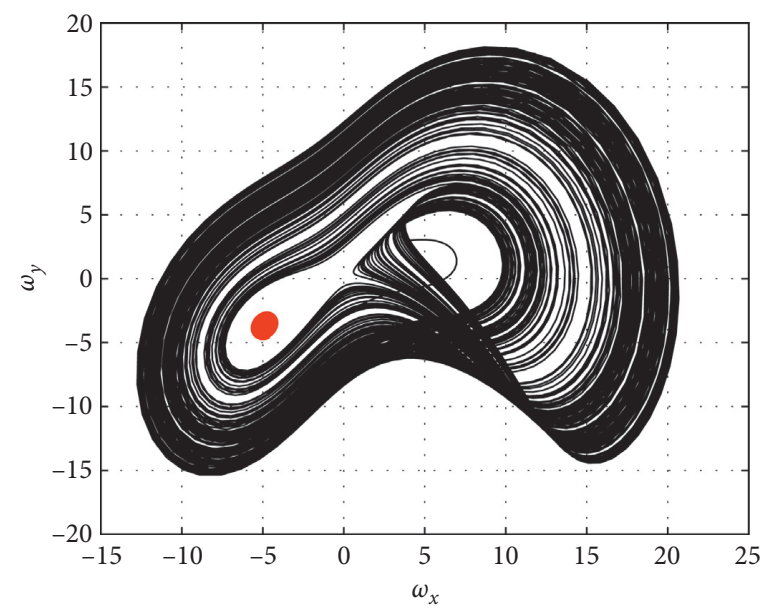

(c)

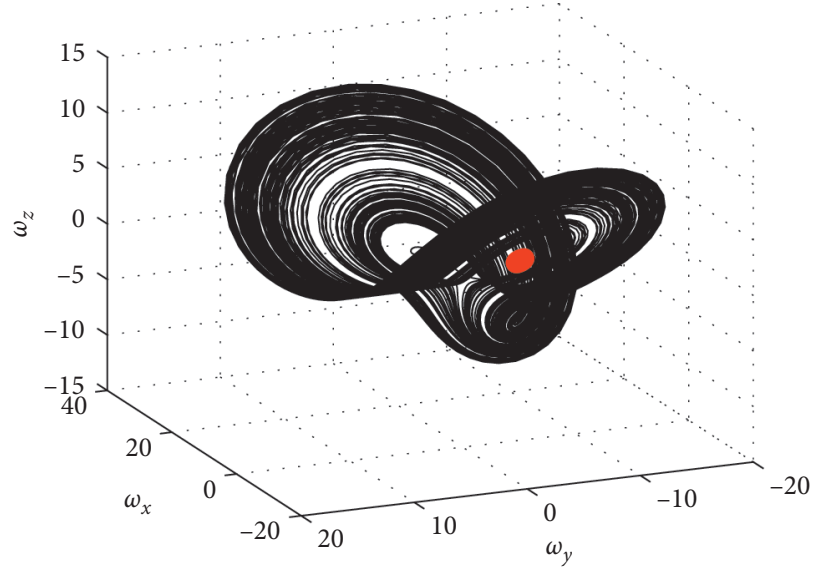

(b)

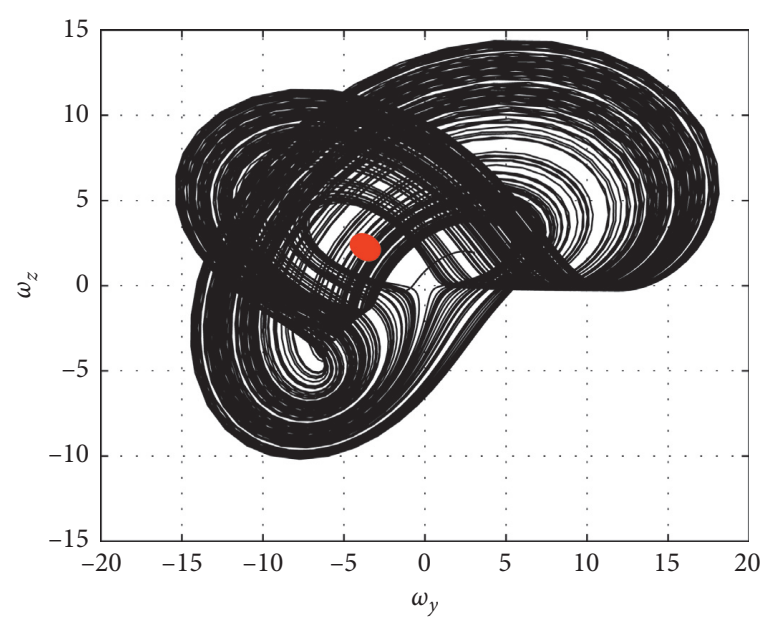

(d)

FIGURE 4: One single-body-three-core-tree-wing chaotic attractor of system (13): (a) attraction plane around S4; (b) chaotic attractor; (c) the projection on the $O \omega_{x} \omega_{y}$ plane; (d) the projection on the $O \omega_{x} \omega_{y}$ plane. 
3.2.4. Multifarious Chaotic Attractors. Keeping $\omega_{u}=0$, changing parameter value of $a_{x}, a_{y}$, and $a_{z}$ and matrix A, system (7) will exhibit chaotic attitude motion, and a lot of new chaotic attractors are found in state space, as follows:

Example 4. $\left(a_{x}, a_{y}, a_{z}\right)=(10,-10,10)$ and $\vec{A}=$

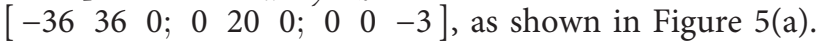

Example 5. $\left(a_{x}, a_{y}, a_{z}\right)=(4,-1,2)$ and $\vec{A}=[-88-2 ; 10-1$ -10.8-3], as shown in Figure 5(b).

Example 6. $\left(a_{x}, a_{y}, a_{z}\right)=(0,-1,1)$ and $\vec{A}=$ $[-77-6 ; 40-1-33 ; 550-3]$, as shown in Figure 5(c).

Example 7. $\left(a_{x}, a_{y}, a_{z}\right)=(1,-1,1)$ and $A=$ $[-660 ; 05-0.8 ; 1010-1]$, as shown in Figure 5(d).

\section{Analysis of the Properties of New Chaotic Attractors}

In order to analyze the structure of new chaotic attractors, the concept of attractive plane is introduced from the linear system:

$$
\frac{\mathrm{d} \vec{x}}{\mathrm{~d} t}=\vec{B} \vec{x}
$$

where $\vec{B} \in R^{3 \times 3}$ is nonsingular coefficient matrix. $\vec{x}=\left[x_{1}, x_{2}, x_{3}\right]^{T}$ is state vector. If the matrix $\vec{B}$ has one real eigenvalue $\lambda_{1}$ and a pair of complex conjugate eigenvalues $\lambda_{2,3}=\alpha \pm \beta i$, the solution of equation (16) can be written as

$$
\begin{aligned}
x_{i}\left(t, x_{i 0}\right) & =C_{i 1} e^{\lambda_{1} t}+C_{i 2} e^{\alpha t} \cos \beta t+C_{i 3} e^{\alpha t} \sin \beta t \\
& =C_{i 1} e^{\lambda_{1} t}+k_{i}^{\prime \prime}\left(t, x_{i 0}\right),
\end{aligned}
$$

where $C_{i j}(i, j=1,2,3)$ are coefficients in relation to structure parameters of system (23) and initial values. $k_{i}^{\prime \prime}\left(t, x_{i 0}\right)$ is the partial solution of (23).

Theorem 1. If the matrix $\vec{B}$ has complex conjugate eigenvalues $\lambda_{2,3}=\alpha \pm \beta i$, there must be a fixed plane across coordinate origin $(0,0,0)$ in the phase space, and the motion trajectory of the partial solution must be inside the fixed plane:

$$
k_{i}^{\prime \prime}\left(t, x_{0}\right)=C_{i 2} e^{\alpha t} \cos \beta t+C_{i 3} e^{\alpha t} \sin \beta t, \quad(i=1,2,3) .
$$

Proof. it is assumed that the motion trajectory of the partial solution $k_{i}^{\prime \prime}\left(t, \vec{x}_{0}\right)$ is inside a plane across coordinate origin $(0,0,0)$ in the phase space, and the normal vector of this plan is $\vec{P}=\left\{p_{1}, p_{2}, p_{3}\right\}$. The point-norm form equation of plane can be expressed as

$$
p_{1} k_{1}^{\prime \prime}+p_{2} k_{2}^{\prime \prime}+p_{3} k_{3}^{\prime \prime}=0
$$

Now, let us prove this plane exists, and its normal vector $\vec{P}$ is constant and has nothing to do with the initial conditions. Put formulas (25) into (26):

$$
\begin{aligned}
& \left(p_{1} C_{12}+p_{2} C_{22}+p_{3} C_{32}\right) e^{\alpha t} \cos \beta t \\
& \quad+\left(p_{1} C_{13}+p_{2} C_{23}+p_{3} C_{33}\right) e^{\alpha t} \sin \beta t=0
\end{aligned}
$$

When $t=0, C_{12}=x_{10}, C_{22}=x_{20}, C_{32}=x_{30}$, and $\dot{x}_{i}(0)=$ $\alpha C_{i 2}+\beta C_{i 3}=\alpha x_{i 0}+\beta C_{i 3}=a_{i 1} x_{10}+a_{i 2} x_{20}+a_{i 3} x_{30}$, then we have

$$
\begin{aligned}
& \left(p_{1} x_{10}+p_{2} x_{20}+p_{3} x_{30}\right) e^{\alpha t} \cos \beta t \\
& \quad+\left[\begin{array}{c}
p_{1}\left(a_{11} x_{10}-\alpha x_{10}+a_{12} x_{20}+a_{13} x_{30}\right) \\
+p_{2}\left(a_{21} x_{10}+a_{22} x_{20}-\alpha x_{20}+a_{23} x_{30}\right) \\
+p_{3}\left(a_{31} x_{10}+a_{32} x_{20}+a_{33} x_{30}-\alpha x_{30}\right)
\end{array}\right] e^{\alpha t} \sin \beta t=0 .
\end{aligned}
$$

As $p_{1} x_{10}+p_{2} x_{20}+p_{3} x_{30}=0$, we have

$$
\begin{aligned}
& \left(p_{1} a_{11}+p_{2} a_{21}+p_{3} a_{31}\right) x_{10}+\left(p_{1} a_{12}+p_{2} a_{22}+p_{3} a_{32}\right) x_{20} \\
& \quad+\left(p_{1} a_{13}+p_{2} a_{23}+p_{3} a_{33}\right) x_{30}=0
\end{aligned}
$$

As long as let $\left(p_{1} a_{11}+p_{2} a_{21}+p_{3} a_{31}\right) / p_{1}=\left(p_{1} a_{12}+\right.$ $\left.p_{2} a_{22}+p_{3} a_{32}\right) / p_{2}=\left(p_{1} a_{13}+p_{2} a_{23}+p_{3} a_{33}\right) / p_{3}=k$, it can be derived as follows:

$$
\left[\begin{array}{ccc}
a_{11}-k & a_{21} & a_{31} \\
a_{12} & a_{22}-k & a_{32} \\
a_{13} & a_{23} & a_{33}-k
\end{array}\right]\left[\begin{array}{l}
p_{1} \\
p_{2} \\
p_{3}
\end{array}\right]=0
$$

where $k$ is the ratio of vectors module. Arbitrarily choose one vector coordinate, for example, $p_{1}=1$, and other coordinates $\left(p_{1}, p_{2}, p_{3}\right)$ could be solved. Obviously, it has nothing to do with the initial conditions and the eigenvalue $\lambda$. Proof ends.

Definition 1. Assume the matrix $\vec{B}$ has one real eigenvalue $\lambda_{1}$ and a pair of complex conjugate eigenvalues $\lambda_{2,3}=\alpha \pm \beta i$. When $\lambda_{1}<0$ (or $\lambda_{1}>0$ ), the fixed plane is named attractive plane (or repulsive plane) in which the partial solution $k_{i}^{\prime \prime}\left(t, \vec{x}_{0}\right)$ is inside.

\subsection{Structural Properties of Chaotic Attractors}

Properties 1. The size of various attractors in equation (7) is determined by the relative positions of all equilibrium points. In other words, the relative distances of all equilibrium points are magnified $k$ times, and the sizes of various attractors are magnified $1 / k$ times synchronously, and the structure and shape remain unchanged.

The parameters $a_{x}, a_{y}$, and $a_{z}$ in equation (7) are simultaneously magnified $k$ times, and the sizes of various attractors are magnified $1 / k$ times, and the structure and shape remain unchanged. This conclusion can be easily drawn from equilibrium calculation formulas (13) and (22) of equations (12) and (21), respectively.

Properties 2. There is an attractive plane in the neighborhood of each equilibrium point in the chaotic system. The nonlinear motion mode near an equilibrium point of a 


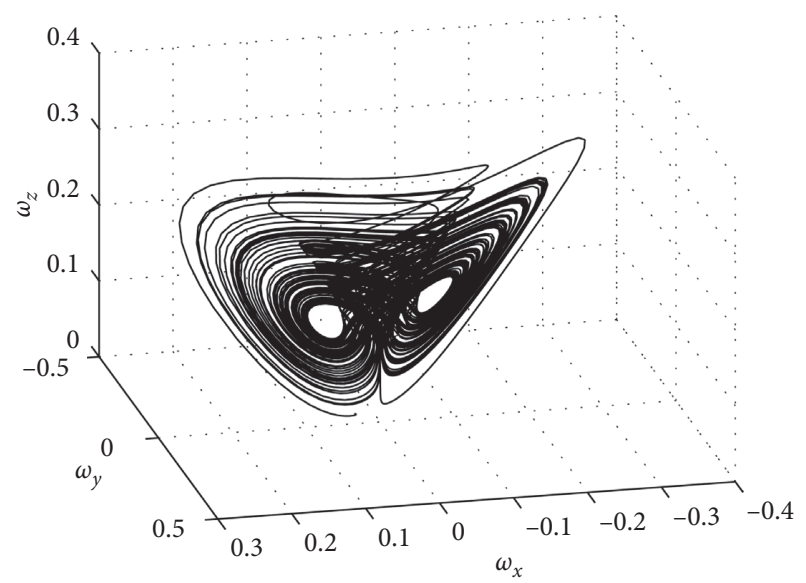

(a)

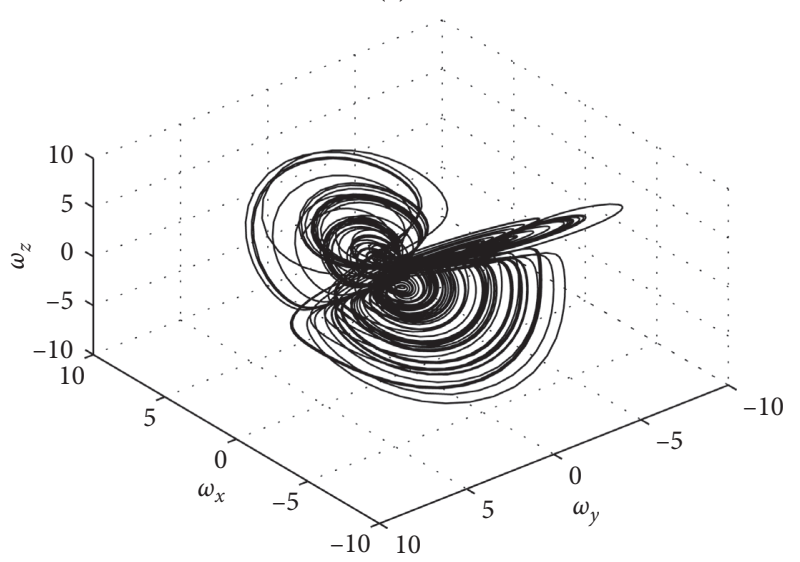

(c)

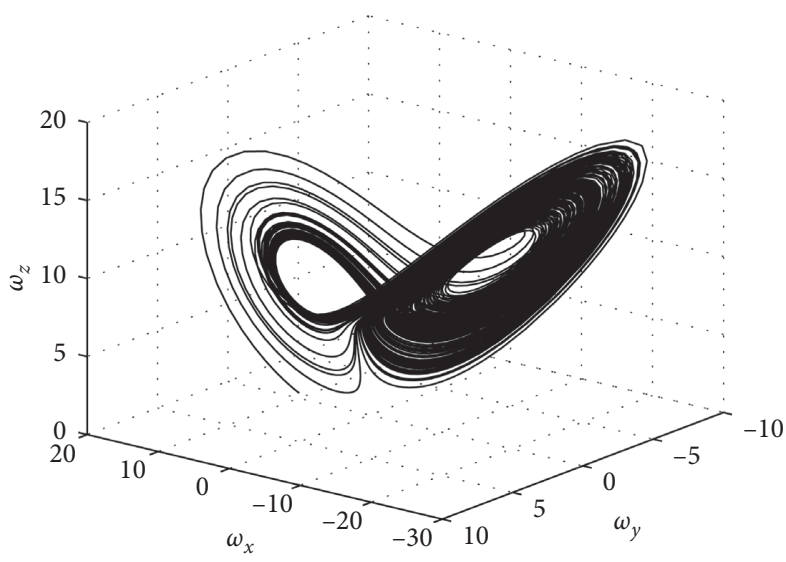

(b)

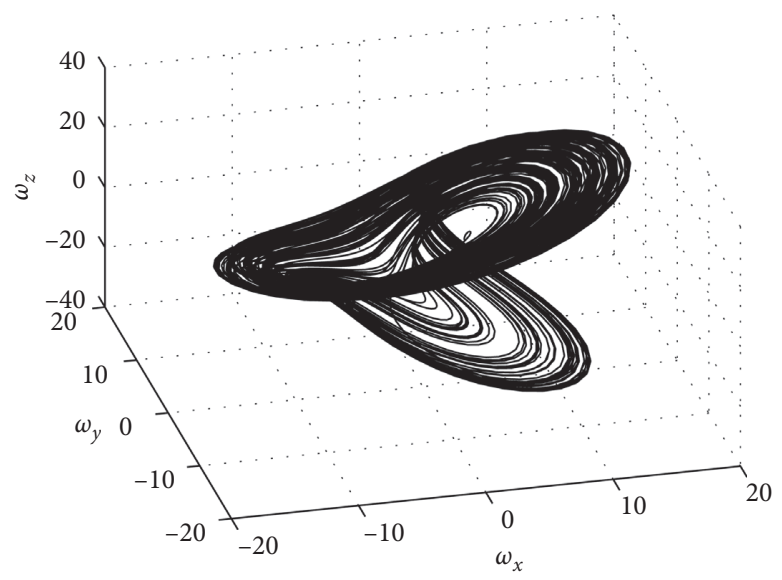

(d)

FiguRE 5: Four new chaotic attractor of system (7): (a) example 4; (b) example 5; (c) example 6; (d) example 7.

complicated chaotic system can be approximately described with its linearized equation. Each chaotic motion comprises at least two nonlinear motion modes. The essential reason to form chaotic attractor is nonperiodic and asynchronous switch between two modes.

\section{Control of Chaotic Attitude Motion in a Spinning Spacecraft}

A spinning spacecraft usually is required to rotate along one axis with constant rotation rate and not rotate along the other two axes. The goal of control is to stabilize the state of the system at the desired equilibrium point $\left(\omega_{x}, \omega_{y}, \omega_{z}\right)=$ $(c, 0,0)$. The angular velocity $\omega$ in system $(7)$ cannot be measured directly in practice and $\omega_{y} \neq \dot{\theta}, \omega_{z} \neq \dot{\psi}$ generally. The attitude stabilization system measures the attitude angle $\phi, \theta$, and $\psi$ and the attitude angular velocities $\dot{\phi}, \theta$, and $\dot{\psi}$ of the spacecraft using attitude sensors such as earth sensors, solar sensors, and gyroscopes. Compared with the desired attitude angle and angular velocity, if there is a deviation, the switch control instruction is formed by the controller to suppress the unwanted rotation of the spacecraft.

As all know, the nonlinear relay control law which is based on position and velocity feedback with dead band peculiarity is suitable for the nonchaotic system when $\vec{A}=0$ in system (7). However, the situation is completely different for chaotic systems, because there are multiple equilibrium points in the system, and the control torques applied to the three axes are interacting. So, the attitude angle change of other direction must be considered into the design when the control law for one direction was designed.

Let us control six-dimensional chaotic system (21) (its chaotic attractor is shown in Figure 5(a)):

$$
\left\{\begin{array}{l}
\dot{\omega}_{x}=\frac{10 \omega_{y} \omega_{z}-36 \omega_{x}+36 \omega_{y}+u_{x}}{I_{x}}, \\
\dot{\omega}_{y}=\frac{-10 \omega_{x} \omega_{z}+20 \omega_{y}+u_{y}}{I_{y}}, \\
\dot{\omega}_{z}=\frac{10 \omega_{y} \omega_{z}-3 \omega_{z}+u_{z}}{I_{z}}, \\
\dot{\varphi}=\frac{\left(\omega_{x} \cos \psi-\omega_{y} \sin \psi\right)}{\cos \theta} \\
\dot{\theta}=\omega_{x} \sin \psi+\omega_{y} \cos \psi, \\
\dot{\psi}=\omega_{z}-\left(\omega_{x} \cos \psi-\omega_{y} \sin \psi\right) \tan \theta .
\end{array}\right.
$$




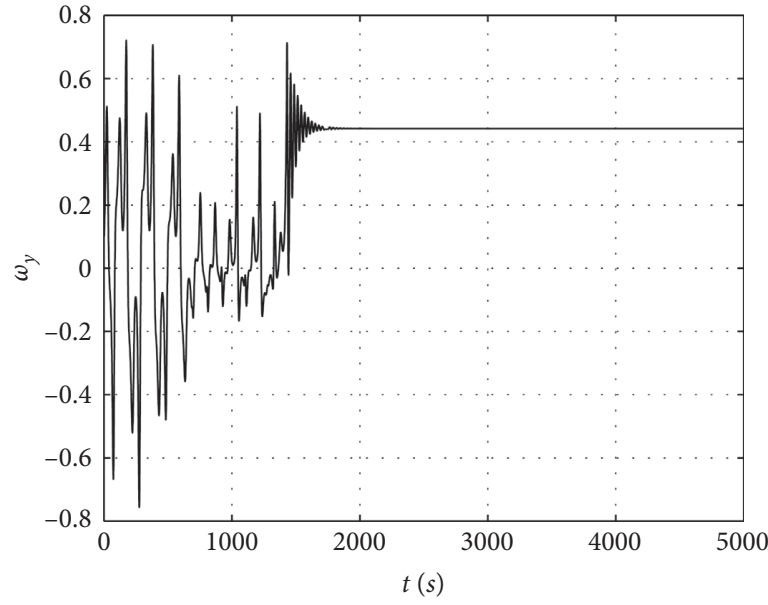

(a)

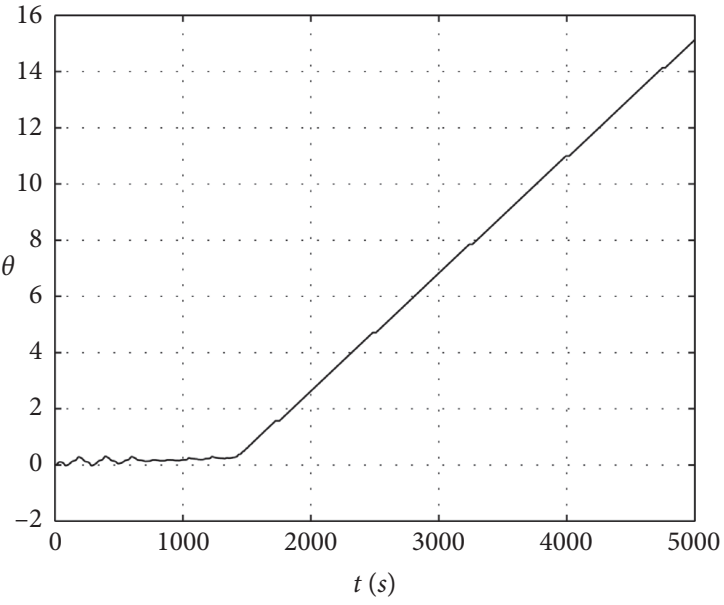

(b)

Figure 6: The time history of $\omega_{y}$ and $\theta$ under single-channel relay control action: (a) the time history of $\omega_{y}$; (b) the time history of $\theta$.

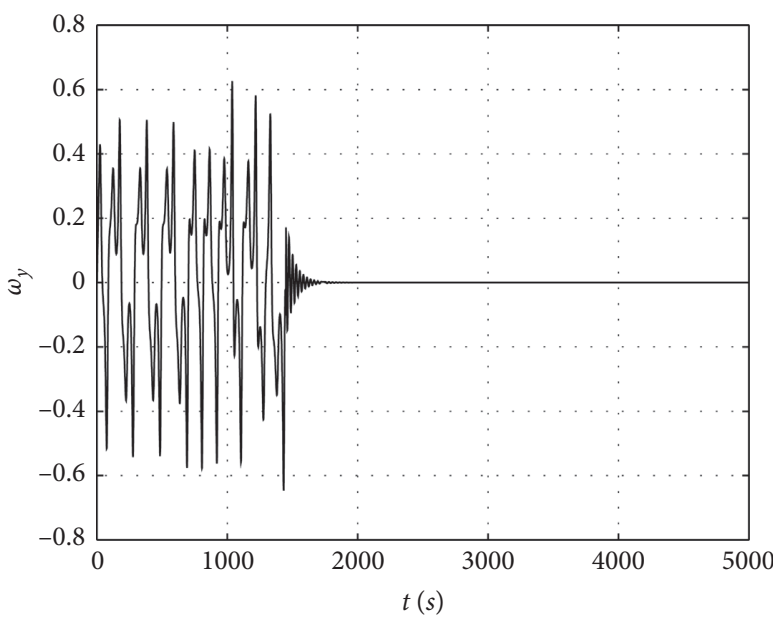

(a)

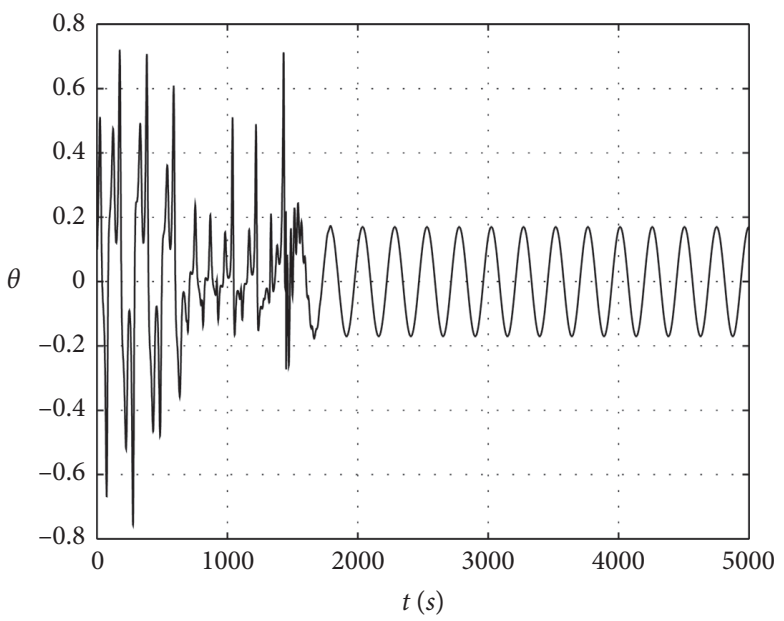

(b)

FIgURE 7: The time history of $\omega_{y}$ and $\theta$ under two-channel relay control action: (a) the time history of $\omega_{y}$; (b) the time history of $\theta$.

Adopting single-channel dead zone relay control law based on position and speed feedback,

$$
\begin{aligned}
& u_{x}(\varphi, \dot{\varphi})= \begin{cases}-I_{x} M, & \text { when } \varphi>\varphi_{1}, \dot{\varphi}>-\dot{\varphi}_{1}, \\
0, & \text { when }|\varphi|<\left|\varphi_{1}\right|,|\dot{\varphi}|>\left|\dot{\varphi}_{1}\right|, \\
I_{x} M, & \text { when } \varphi<\varphi_{1}, \dot{\varphi}<\dot{\varphi}_{1},\end{cases} \\
& u_{y}(\theta, \dot{\theta})= \begin{cases}-I_{y} M, & \text { when } \theta>\theta_{1}, \dot{\theta}>-\dot{\theta}_{1}, \\
0, & \text { when }|\theta|<\left|\theta_{1}\right|,|\dot{\theta}|>\left|\dot{\theta}_{1}\right|, \\
I_{y} M, & \text { when } \theta<\theta_{1}, \dot{\theta}<\dot{\theta}_{1},\end{cases} \\
& u_{z}(\psi, \dot{\psi})= \begin{cases}-I_{z} M, & \text { when } \psi>\psi_{1}, \dot{\psi}>-\dot{\psi}_{1}, \\
0, & \text { when }|\psi|<\left|\psi_{1}\right|,|\dot{\psi}|>\left|\dot{\psi}_{1}\right|, \\
I_{z} M, & \text { when } \psi<\psi_{1}, \dot{\psi}<\dot{\psi}_{1},\end{cases}
\end{aligned}
$$

where $M$ is control torque to be generated by nozzle thrusters. $\varphi_{1}, \theta_{1}, \psi_{1}$ is angle position error of dead band corresponding to the driving signal when the nozzle baffle is opened. There are five equilibriums in equations (21), and the point $S_{1}$ : $(-0.34,0.44,0.34)$ is one of five equilibriums. The control action may drive any state to approach a nearest equilibrium point from an initial state. An equilibrium state is not the desired state for a spinning spacecraft though the chaotic motion is suppressed. Figure 6 shows the time history of $\omega_{y}$ and $\theta$ under the single-channel dead zone relay control action, starting from initial state $(0.2,0.1,0.2)$, beginning to control on $1500 \mathrm{~s}$, and ending in the equilibrium point $S_{1}:(-0.34,0.44,0.34)$.

Now, considering the mutual effect among $\omega_{x}, \omega_{y}$, and $\omega_{z}$, changing the dual-channel control law to be 


$$
\begin{aligned}
& u_{y}= \begin{cases}-I_{y} M, & \text { when } \theta>\theta_{1}, \dot{\theta}>-\dot{\theta}_{1}, \text { and } \varphi<\varphi_{1}, \dot{\varphi}<\dot{\varphi}_{1}, \\
I_{y} M, & \text { when } \theta>\theta_{1}, \dot{\theta}>-\dot{\theta}_{1} \text {, and } \varphi>\varphi_{1}, \dot{\varphi}>-\dot{\varphi}_{1}, \\
0, & \text { when }|\theta|<\left|\theta_{1}\right|,|\dot{\theta}|>\left|\dot{\theta}_{1}\right| \text {, and }|\varphi|<\left|\varphi_{1}\right|,|\dot{\varphi}|<\left|\dot{\varphi}_{1}\right|, \\
-I_{y} M, & \text { when } \theta<\theta_{1}, \dot{\theta}<\dot{\theta}_{1}, \text { and } \varphi<\varphi_{1}, \dot{\varphi}<\dot{\varphi}_{1}, \\
I_{y} M, & \text { whan } \theta<\theta_{1}, \dot{\theta}<\dot{\theta}_{1}, \text { and } \varphi>\varphi_{1}, \dot{\varphi}>-\dot{\varphi}_{1},\end{cases} \\
& u_{x}= \begin{cases}+I_{x} M, & \text { when } \theta>\theta_{1}, \dot{\theta}>-\dot{\theta}_{1}, \text { and } \varphi<\varphi_{1}, \dot{\varphi}<-\dot{\varphi}_{1}, \\
-I_{x} M, & \text { when } \theta>\theta_{1}, \dot{\theta}>-\dot{\theta}_{1}, \text { and } \varphi>\varphi_{1}, \dot{\varphi}>-\dot{\varphi}_{1}, \\
0, & \text { when }|\theta|<\left|\theta_{1}\right|,\left|\dot{\theta}_{\mid}\right| \dot{\theta}_{1} \mid, \text { and }|\varphi|<\left|\varphi_{1}\right|,|\dot{\varphi}|>\left|\dot{\varphi}_{1}\right|, \\
+I_{x} M, & \text { when } \theta<\theta_{1}, \dot{\theta}<\dot{\theta}_{1}, \text { and } \theta>\theta_{1}, \dot{\theta}>\dot{\theta}_{1}, \\
-I_{x} M, & \text { when } \theta<\theta_{1}, \dot{\theta}<\dot{\theta}_{1}, \text { and } \theta<\theta_{1}, \dot{\theta}<\dot{\theta}_{1} .\end{cases}
\end{aligned}
$$

Figure 7 shows the time history of $\omega_{y}$ and $\theta$ under the two-channel control action: initial state $(0.2,0.1,0.2)$; end point $(3,0,0)$.The control effect meets the desired operating requirements of a spinning spacecraft with constant rotation rate.

\section{Conclusion}

The Euler dynamical equation which describes the attitude motion of a rigid body will exhibit very complex dynamic behaviors and include many special types of chaotic attractors. In the same phase space, beyond chaotic attractors, there may be a very large hidden attractor or very small hidden attractors. Even if the system is stable near a certain equilibrium point, chaotic attractors may still be generated in a larger space. From the simulation analysis, we can see that the stability of the linearized equation near the equilibrium point determines the shape and size of the chaotic attractor. The single-channel dead zone relay control method cannot effectively suppress a chaotic motion of spinning spacecraft. The simulation results confirm that the single-channel dead zone relay control method cannot effectively suppress a chaotic motion of spinning spacecraft. The dual-channel control method proposed in this paper can control a chaotic system to an appointed equilibrium point or a periodic orbit.

\section{Data Availability}

All the data generated or analyzed during this study are included in this article.

\section{Conflicts of Interest}

The authors declare that they have no conflicts of interest.

\section{Acknowledgments}

This work was supported by the Natural Science Basic Research Plan in Shaanxi Province of China (2020JM-646), the Innovation Capability Support Program of Shaanxi
(2018GHJD-21), the Science and Technology Program of Xi' an (2019218414GXRC020CG021-GXYD20.3), the Support Plan for Sanqin Scholars Innovation Team in Shaanxi Province of China, the Special fund for high-level talents of Xijing University (XJ20B07), the Key Research and Development Program of Shaanxi (No. 2019GY-025), and Xi'an Science and Technology Plan Project (2020KJRC0134).

\section{References}

[1] P. A. Meehan and S. F. Asokanthan, "Control of chaotic instabilities in a spinning spacecraft with dissipation using lyapunov's method," Chaos, Solitons \& Fractals, vol. 13, no. 9, pp. 1857-1869, 2002.

[2] Y. Meng, R. Hao, and Q. Chen, "Attitude stability analysis of a dual-spin spacecraft in halo orbits," Acta Astronautica, vol. 99, pp. 3118-3129, 2014.

[3] M. Nazari and E. A. Butcher, "On the stability and bifurcation analysis of dual-spin spacecraft," Acta Astronautica, vol. 93, pp. 162-175, 2014.

[4] A. I. Neishtadt and M. L. Pivovarov, "Separatrix crossing in the dynamics of a dual-spin satellite," Journal of Applied Mathematics and Mechanics, vol. 64, no. 5, pp. 709-714, 2000.

[5] A. V. Doroshin, "Homoclinic solutions and motion chaotization in attitude dynamics of a multi-spin spacecraft," Communications in Nonlinear Science and Numerical Simulation, vol. 19, pp. 2528-2552, 2014.

[6] A. V. Doroshin, "Initiations of chaotic regimes of attitude dynamics of multi-spin spacecraft and gyrostat-satellites basing on multiscroll strange chaotic attractors," in Proceedings of the SAI Intelligent Systems Conference (IntelliSys), pp. 698-704, London, UK, November 2015.

[7] A. V. Doroshin, "Multi-spin spacecraft and gyrostats as dynamical systems with multiscroll chaotic attractors," in Proceedings of the SAI Intelligent Systems Conference Conference (SAI), pp. 882-887, Coleraine, Northern Ireland, October 2014.

[8] V. S. Aslanov and A. V. Doroshin, "Chaotic dynamics of an unbalanced gyrostat," Journal of Applied Mathematics and Mechanics, vol. 74, pp. 525-560, 2010.

[9] J. L. Kuang, S. H. Tan, K. Arichandan, and A. Y. T. Leung, "Chaotic attitude motion of gyrostat satellite via melnikov method," International Journal of Bifurcation and Chaos in Applied Sciences and Engineering, vol. 11, pp. 1233-1260, 2006.

[10] J. Kuang, S. Tan, K. Arichandran, and A. Y. T. Leung, "Chaotic dynamics of an asymmetrical gyrostat," International Journal of Non-linear Mechanics, vol. 36, no. 8, pp. 1213-1233, 2001.

[11] A.V. Doroshin, "Modeling of chaotic motion of gyrostats in resistant environment on the base of dynamical systems with strange attractors," Communications in Nonlinear Science and Numerical, vol. 16, no. 8, pp. 3188-3202, 2011.

[12] K. H. Shirazi and M. H. Ghaffari-Saadat, "Chaotic motion in a class of asymmetrical Kelvin type gyrostat satellite," International Journal of Non-linear Mechanics, vol. 39, no. 5, pp. 785-793, 2004.

[13] X. Tong and B. Tabarrok, "Chaotic motion of an asymmetric gyrostat in the gravitational field," International Journal of Non-Linear Mechanics, vol. 30, no. 3, pp. 191-203, 1995.

[14] V. V. Beletsky and R. V. F. Lopes, M. L. Pivovarov, Chaos in spacecraft attitude motion in earth's magnetic field," Chaos: An Interdisciplinary Journal of Nonlinear Science, vol. 9, no. 2, pp. 493-498, 1999. 
[15] L. Yan-Zhu, J. Yu Hong, and C. Li-Qun, "Chaotic attitude motion and its control of spacecraft in elliptic orbit and geomagnetic field," Acta Astronautica, vol. 55, pp. 487-494, 2004.

[16] W. Liu, G. Chen, A new chaotic system and its generation," International Journal of Bifurcation and Chaos, vol. 13, no. 1, pp. 261-267, 2003.

[17] R. B. Leipnik and T. A. Newton, "Double strange attractors in rigid body motion with linear feedback control," Physics Letters A, vol. 86, pp. 63-70, 1981.

[18] L.-Y. Kong, F.-Q. Zhou, and J. Zou, "The control of chaotic attitude motion of a perturbed spacecraft," in Proceedings of the 2006 Chinese Control Conference, Harbin, China, August 2006.

[19] L.-Y. Kong and Y.-Y. Fan, "New chaotic attractors and sliding mode control of rigid-body rotation dynamical system," in Proceedings of the 2010 Chinese Control and Decision Conference, Xuzhou, China, November 2010.

[20] D. I. Martinez, J. J. De Rubio, T. M. Vargas, V. Garcia et al., "Stabilization of robots with a regulator containing the sigmoid mapping," IEEE Access, vol. 8, pp. 89479-89488, 2020.

[21] J. Humberto and A. T. M. Pedro, "Exponential synchronization of chaotic xian system using linear feedback control," Complexity, vol. 2019, Article ID 4706491, 10 pages, 2019.

[22] J. D. J. Rubio, G. Ochoa, D. Mujica-Vargas et al., "Structure regulator for the perturbations attenuation in a quadrotor," IEEE Access, vol. 7, pp. 138244-138252, 2019.

[23] A. I. Carlos, "A trajectory planning based controller to regulate an uncertain 3D overhead crane system," International Journal of Applied Mathematics and Computer Science, vol. 29-4, pp. 693-702, 2019.

[24] J. O. Escobedo-Alva, E. C. Garcia-Estrada, L. A. ParamoCarranza, J. A. Meda-Campana, and R. Tapia-Herrera, "Theoretical application of a hybrid observer on altitude tracking of quadrotor losing GPS signal," IEEE Access, vol. 6, pp. 76900-76908, 2018.

[25] J. D. J. Rubio, D. I. Martinez, V. Garcia, G. J. Gutierrez et al., "The perturbations estimation in two gas plants," IEEE Access, vol. 8, pp. 83081-83091, 2020.

[26] P. M. Alban and A. J. Jones, "The control of higher dimensional chaos: comparative results for the chaotic satellite attitude control problem," Physica D, vol. 135, pp. 41-46, 2000. 